www.jmscr.igmpublication.org

Impact Factor 5.84

Index Copernicus Value: 83.27

ISSN (e)-2347-176x ISSN (p) 2455-0450

crossref DOI: https://dx.doi.org/10.18535/jmscr/v5i2.38

\begin{abstract}
Journal Of Medical Science And Clinical Research
IGM Publication

An official Publication of IGM Publication
\end{abstract}

\title{
Suppurative BCG Lymphadenitis in an Infant
}

\author{
Authors \\ Dr. Dipak Sonawane ${ }^{1}$, Dr. Sushant Mane ${ }^{2}$, Dr. Muneshwar Bhongade ${ }^{3}$, \\ Dr. Karthik Kota ${ }^{4}$, Dr. Bushra Ansari ${ }^{5}$ \\ ${ }^{2}$ Associate Professor, ${ }^{1,3,4,5}$ Residents
}

Department of Pediatrics, Grant Government Medical College \& Sir JJ Group of Hospitals, Mumbai

Corresponding Author

Dr. Dipak Sonawane

Resident, Department Of Pediatrics,

Grant Government Medical College \& Sir JJ Group of Hospitals, Mumbai, INDIA

\begin{abstract}
Introduction: Tuberculosis is a major cause of morbidity and mortality in developing countries including India. BCG vaccination is one of the important measures to decrease serious forms of tuberculosis i.e. military or disseminated tuberculosis especially in the pediatric age group. BCG vaccination was first included in the World Health Organization's "Expanded Programme on Immunisation" in 1974. It is considered to be a safe vaccine as it has a very low incidence of serious side effects. Though the incidence of side effects is low, the mass administration means there are some effects which are bound to occur. BCGLymphadenitis is one such unusual complication. There are two types of BCG-lymphadenitis. Simple lymphadenitis and suppurative lymphadenitis. Simple BCG lymphadenitis usually doesn't require any treatment and resolve on its own over a period of few weeks. Suppurative lymphadenitis on the other hand is characterised by appearance of erythema and other signs of inflammation over the skin overlying affected lymph nodes. Clinically fluctuation may be present in the swelling. The treatment of BCG adenitis has been controversial. In cases of simple lymphadenitis nothing is required and the adenitis is expected to resolve on its own in next few weeks. But in cases where the size of lymph node is more than $1.5 \mathrm{~cm}$ and there is tenderness, fluctuation, signs of inflammation or sinus formation, antitubercular drugs are indicated.

Case Report: A 9 months old male child was brought to us with a history of gradually increasing swelling in left axillary region since 3 months. The swelling ruptured 10 days back draining a pus-like material. There was history of BCG vaccination to the child 3 months prior to the appearance of the swelling. There was no family history of tuberculosis. On examination, the baby was healthy and playful. BCG scar was present on left deltoid region. There was a non tender, fluctuant swelling with discharge in the left axillary region. The child was initially treated with oral Amoxicillin-Clavulanate but had no improvement. In view of history of BCG vaccination and classical clinical findings a provisional diagnosis of suppurative BCG lymphadenitis was made. Routine investigations and chest $x$ ray were normal. USG of left axilla showed multiple enlarged necrotic lymph nodes. Left axillary lymph node biopsy was done. Biopsy specimen didn't show Acid Fast Bacilli on smear, but MTB was detected with no resistance to Rifampicin on Gene Xpert MTB RIF and Mycobacterium Tuberculosis Complex was isolated on culture. Category 1 antitubercular therapy was started for 6 months. Baby promptly responded to antitubercular treatment and was discharged
\end{abstract}


with an advice to complete the prescribed treatment.

Conclusion: $B C G$ is usually considered a safe vaccine. Side effects following BCG vaccination are unusual but may occur. Non-suppurative BCG adenitis regresses spontaneously and usually no treatment is required. While suppurative lymphadenitis may require management in the form of antitubercular therapy. Immunodeficiency disorders should be excluded in children having severe suppurative lymphadenitis following BCG vaccination. Education and awareness of this entity in patients and paramedical personnel like anganwadi workers who are primarily involved in BCG vaccination in rural areas is essential to diagnose and treat this unusual complication of BCG vaccination

Keywords: BCG Adenitis, Gene Xpert, Antitubercular Treatment.

\section{Introduction}

BCG vaccine has been in use for prevention of tuberculosis since 1921. The other use of this vaccine is for immunostimulation in neoplasia. It has been included in World health organisation's expanded program of immunisation since $1974^{[1]}$. Though the efficacy of BCG in preventing tuberculosis is a controversial preposition it has been found to be effective in prevention of severe form of tuberculosis [2]. The efficacy rates determined in various studies are so diverse that it has been estimated to be in between $0-80 \%{ }^{[3]}$. The controversies regarding efficacy of BCG vaccine in prevention of primary tuberculosis is due to different results in various studies. The possible explanations of different efficacy rates were found to be differences in latitude, vaccine strain, and methodological differences ${ }^{[4]}$. Not withstanding these controversies the efficacy of this vaccine is well established in prevention of serious forms of tuberculosis in immunocompetent children ${ }^{[5]}$. For this reason it has been included in various immunization programmes including Universal Immunisation Programme (UIP) and Expanded Programme on Immunisation $(\mathrm{EPI})^{[6]}$. BCG vaccine is considered a safe vaccine and adverse effects are usually local including local erythema, induration, papule formation, abscess and discharging sinus. Regional lymphadenopathy involving the axillary lymph nodes may occur. In rare instances cervical lymph nodes may also get involved ${ }^{[7]}$. Systemic complications like soft tissue granulomas, osteomyelitis and disseminated disease are extremely rare. Children with acquired immunodeficiency syndrome (AIDS) or conge- nitalimmuno deficient conditions are predisposed for systemic complications of BCG vaccination ${ }^{[8]}$. The lymphadenitis after BCG vaccination can be simple or suppurative. The simple lymphadenitis following BCG vaccination usually doesn't require any treatment except local hygiene and is expected to resolve spontaneously ${ }^{[9]}$. The suppurative form of BCG adenitis is characterised by enlargement of regional lymph nodes and suppuration. Clinically it can be recognised by fluctuant swelling. In majority of the cases the overlying skin shows subcutaneous edema, redness and pustule formation, if not treated properly. If left untreated, the suppuration will rupture causing caseous discharge and sinus formation. The treatment of suppurative lymphadenitis consist of proper diagnosis and antitubercular treatment ${ }^{[10]}$.

\section{Case Report}

A 9 months old male child was brought to us with a history of gradually increasing swelling in left axillary region since 3 months which ruptured 10 days back draining a pus-like material. Birth history revealed he was a full term child born by normal vaginal delivery. Developmentally baby was normal and achieved gross motor, fine motor, language, social and adaptive milestones appropriate for his age. He was immunised with BCG and first dose of OPV, DPT, HBV at the age of 3 months followed by second and third dose of OPV, DPT and HBV vaccines at 4 and 5 months of age. He had also received measles vaccine. There was no history of Kochs contact. On examination BCG scar was noted on left deltoid region [Fig: 1]. 


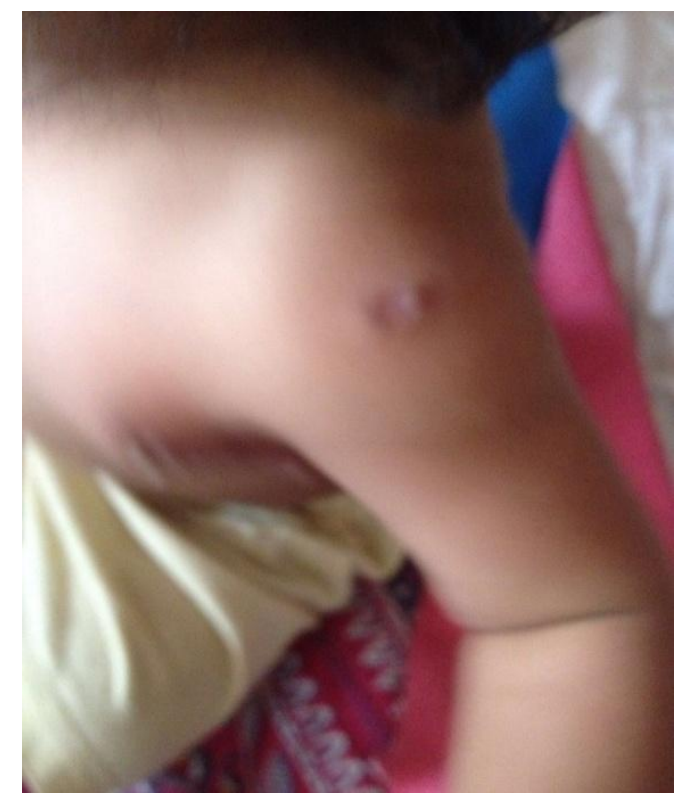

Figure- 1: BCG scar seen on left deltoid region of the baby.

Baby was healthy and playful. Left axillary region showed a round, non-tender fluctuant swelling of $2.5 \mathrm{~cm}$ size. The overlying skin was mildly erythematous and pus could be seen discharging from the site of lymphadenopathy [Fig: 2].

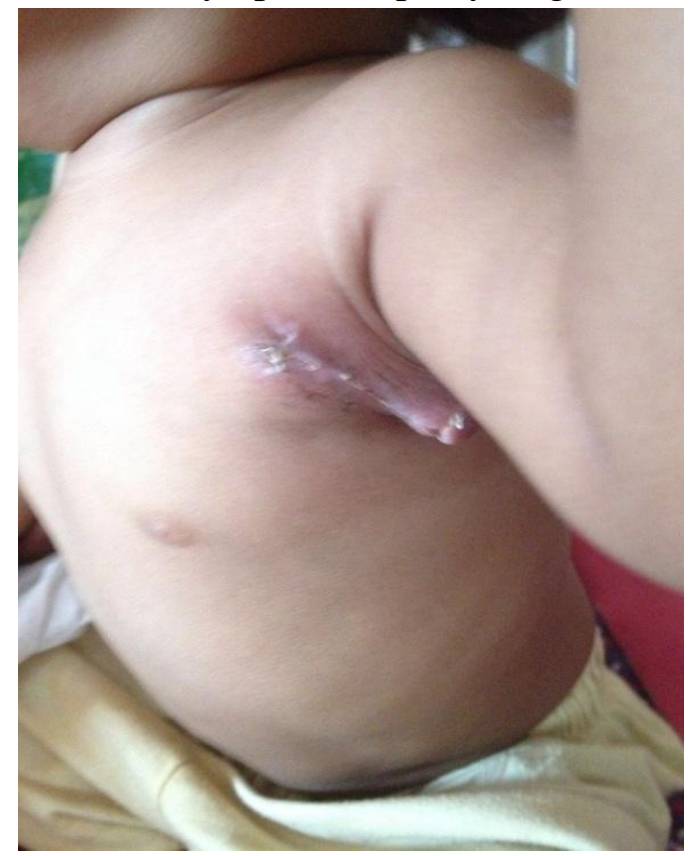

Figure-2: Axillary lymphadenopathy with discharging pus.

Routine hematological investigations and chest Xray were normal. Baby was found to be seronegative for HIV test. Ultrasound of the swelling revealed multiple hypo-echoic necrotic lymph nodes. In view of history of BCG vaccination and classical clinical findings a provisional diagnosis of suppurative BCG lymphadenitis was made. Left axillary lymph node biopsy was done. Smear examination of the biopsy specimen didn't show any acid fast bacilli but culture of the biopsy specimen by Bactec MGIT 960 TB system showed growth of mycobacterium tuberculosis complex.

Mycobacterium Tuberculosis was detected on Gene XPERT MTB RIF with no resistance to Rifampicin. Depending upon the report of Gene XPERT MTB RIF and Bactec MGIT culture a final diagnosis of BCG adenitis was made and category I antitubercular treatment was started. The patient responded well to antitubercular treatment with a reduction in size of the swelling and hence he was discharged with an advice to complete the prescribed treatment for 6 months.

\section{Discussion}

BCG vaccine is recommended to be given along with OPV at first contact of a newborn. It is used globally for prevention of serious forms of tuberculosis like tubercular meningitis and disseminated tuberculosis ${ }^{[11]}$. After intradermal injection at the insertion of deltoid the live attenuated strain of BCG gets disseminated to various organs of the body including lymph nodes and causes asymptomatic subclinical lymphadenopathy. This lymphadenopathy is of no clinical significance and does not require any intervention as it is expected to resolve on its own over a matter of few weeks. The usual sites for lymphnodes involvement are ipsilateralaxillary as well as supraclavicular lymph nodes ${ }^{[12]}$. The time of occurrence and resolution in these cases is typical and occurs around 6 weeks after vaccination and leads to suppuration in 3 months and invariably resolve within 6 months ${ }^{[13]}$. Other than this form of lymphadenopathy which is similar to natural tubercular infection and is essential for development of immunity there are 2 other forms of lymphadenitis which can be seen after BCG vaccination. They are non-suppurative simple lymphadenitis and suppurative lymphadenitis. The 
non suppurative form of BCG lymphadenitis is expected to heal on its own and may not require anything. While the other form, suppurative lymphadenitis is characterised clinically by fluctuations in the swelling and erythema and edema of overlying skin ${ }^{[14]}$. The initial diagnosis is usually clinical and characterised by lymphadenitis of unilateral axillary or supraclavicular or a combination of both on the same side of BCG vaccination. There is no fever or constitutional symptoms as seen in pyogenic lymphadenitis. The risk factors for suppurative lymphadenitis are immunocompromised recipient, reactogenic strains and inadvertent injection of large dose or subcutaneous injection of the vaccine ${ }^{[15]}$. The diagnosis of BCG lymphadenitis can be done by biopsy followed by culture or Gene XPERT. The simple BCG adenitis usually doesn't require anything. In cases of suppurative lymphadenitis the treatment usually require wound care and prevention of sinus formation. In patients who have rapidly growing size of lymph-nodes, sinus formation and pus discharge, antitubercular drugs may be required.

\section{Conclusion}

We reported this case because a definitive diagnosis was made by culture using Bactec and Gene XPERT and lymphadenitis responded well to antitubercular treatment. As BCG vaccine in majority of the cases is administered by paramedical workers working in rural areas (Anganwadi workers, ASHA, ANMs) awareness about this entity in primary care giver is of utmost importance. Referral centers, tertiary care hospitals and medical institutes can play a pivotal role in creating awareness of this entity in medical and paramedical staff related to immunisation.

\section{References}

1. Lugosi L. Theoretical and methodological aspects of BCG vaccine from the discovery of Calmette and Guérin to molecular biology. A review. Tuber Lung Dis 1992;73:252-61.
2. Thilothammal N, Krishnamurthy PV, Runyan DK, Banu K. Does BCG vaccine prevent tuberculous meningitis? Archives of Disease in Childhood. 1996;74(2):144147.

3. Ten Dam BG, Toman K, Hitze KL, Guld J. Present knowledge of immunization against tuberculosis. Bull WHO 1976; 54: 255-262.

4. Clemens JD, Chuong JJ, Feinstein AR. The BCG controversy.A methodological and statistical reappraisal. JAMA 1983; 249:2362-9.

5. Awasthi S, Moin S. Effectiveness of BCG vaccination against tuberculous meningitis. Indian Pediatr. 1999 May;36 (5):45560.

6. Basu RN. Expanded programme on immunization and primary health care. $\mathbf{J}$ Commun Dis. 1982 Sep;14(3):183-8. .

7. Asano S. Granulomatous lymphadenitis. J Clin Exp Hematop. 2012;52(1):1-16.

8. Arvas A. Vaccination in patients with immunosuppression. Turkish Archives of Pediatrics/Türk Pediatri Arşivi. 2014;49 (3):181-185.

9. Goraya J, Virdi V. BacilleCalmette-Guérin lymphadenitis. Postgraduate Medical Journal. 2002;78(920):327-329.

10. Guld J, Magnus K, Tolderlund K, BieringSørensen K, Edwards PQ. Suppurative Lymphadenitis following Intradermal B.C.G. Vaccination of the Newborn. British Medical Journal. 1955;2(4947): 1048-1054.

11. Rodrigues LC, Diwan VK, Wheseler JG. Protective effect of BCG against tuberculous meningitis and miliary tuberculosis: a meta-analysis. Int J Epidemiol. 1993 Dec;22(6):1154-8.

12. Behjati M, Ayatollahi J. Post BCG lymphadenitis in vaccinated infants in Yazd, Iran. Iran J Pediatr 2008;18:351-6. 
13. Ustevdt HJ. Local reactions in BCG vaccination. Bull World Health Organ 1950;2:441-68.

14. WHO Tuberculosis Research Office. Suppurative lymphadenitis following intradermal BCG vaccination of preschool children.Bulletin of the World Health Organization. 1955;12(1-2): 143167.

15. Norouzi S, Aghamohammadi A, Mamishi S, Rosenzweig SD, Rezaei N. Bacillus CalmetteGuérin (BCG) complications associated with primary immunodeficiency diseases. The Journal of infection. 2012;64(6):543-554. 